\title{
A POSITIVE SOLUTION FOR SINGULAR DISCRETE BOUNDARY VALUE PROBLEMS WITH SIGN-CHANGING NONLINEARITIES
}

\author{
HAISHEN LÜ, DONAL O’REGAN, AND RAVI P. AGARWAL
}

Received 17 February 2005; Revised 1 March 2005; Accepted 2 March 2005

This paper presents new existence results for the singular discrete boundary value problem $-\Delta^{2} u(k-1)=g(k, u(k))+\lambda h(k, u(k)), k \in[1, T], u(0)=0=u(T+1)$. In particular, our nonlinearity may be singular in its dependent variable and is allowed to change sign.

Copyright (c) 2006 Hindawi Publishing Corporation. All rights reserved.

\section{Introduction}

Let $a, b(b>a)$ be nonnegative integers. We define the discrete interval $[a, b]=\{a, a+$ $1, \ldots, b\}$. All other intervals will carry its standard meaning, for example, $[0, \infty)$ denotes the set of nonnegative real numbers. The symbol $\Delta$ denotes the forward difference operator with step size 1 , that is, $\Delta u(k)=u(k+1)-u(k)$. Furthermore for a positive $m, \Delta^{m}$ is defined as $\Delta^{m} u(k)=\Delta^{m-1}(\Delta u(k))$. In this paper, we will study positive solutions of the second-order discrete boundary value problem

$$
\begin{gathered}
-\Delta^{2} u(k-1)=g(k, u(k))+\lambda h(k, u(k)), \quad k \in[1, T], \\
u(0)=0=u(T+1),
\end{gathered}
$$

where $\lambda>0$ is a constant and $T>2$ is a positive integer. Here, $g:[1, T] \times(0, \infty) \rightarrow \mathbb{R}$ and $h:[1, T] \times[0, \infty) \rightarrow[0, \infty)$ are continuous. As a result, our nonlinearity may be singular at $u=0$ and may change sign.

By a solution $u$ of the boundary value problem $(1.1)$, we mean $u:[0, T+1] \rightarrow \mathbb{R}, u$ satisfies the difference equation (1.1) on $[1, T]$ and the stated boundary data.

We will let $C[0, T+1]$ denote the class of map $u$ continuous on $[0, T+1]$ (discrete topology), with norm $|u|_{\infty}=\max _{k \in[0, T+1]}|u(k)|$.

\section{Main results}

The main result of the paper is the following. 
2 Singular discrete BVPs

Theorem 2.1. Suppose the following conditions hold:

(G) there exist $g_{i}:[1, T] \times(0, \infty) \rightarrow(0, \infty)(i=1,2)$ continuous functions such that

$$
\begin{aligned}
& g_{i}(k, \cdot) \text { is strictly decreasing for } k \in[1, T], \\
& -g_{1}(k, u) \leq g(k, u) \leq g_{2}(k, u) \quad \text { for }(k, u) \in[1, T] \times(0, \infty), \\
& \int_{0}^{1} g_{1}(k, s) d s<\infty \quad \text { for } k \in[1, T], \\
& \forall s_{0}>0, \quad \sup _{s_{0} \leq s}\left|\frac{\partial}{\partial s} g_{2}(\cdot, s)\right| \in C[1, T]
\end{aligned}
$$

$(\mathrm{H})$ there exist $h_{i}:[1, T] \times[0, \infty) \rightarrow(0, \infty)(i=1,2)$ continuous functions such that

$$
\begin{aligned}
& h_{i}(k, \cdot) \text { increasing for } k \in[1, T], \\
& h_{1}(k, u) \leq h(k, u) \leq h_{2}(k, u) \quad \text { for }(k, u) \in[1, T] \times(0, \infty), \\
& \lim _{u \rightarrow \infty} \frac{h_{2}(k, u)}{u}=0 \quad \text { for } k \in[1, T], \\
& \text { there exists } \bar{s}>0 \text { such that } h_{1}(k, \bar{s})>0 \text { for all } k \in[1, T] .
\end{aligned}
$$

Then there exists $\lambda_{0} \geq 0$ such that for every $\lambda \geq \lambda_{0}$, problem (1.1) has at least one solution $u \in C[0, T+1]$ and $u(k)>0$ for $k \in[1, T]$. Moreover, there exists $c_{i}=c_{i}\left(\lambda, g, h, \phi_{1}\right)>0$ $(i=1,2)$ such that

$$
c_{1} \phi_{1}(k) \leq u(k) \leq c_{2}\left(\phi_{1}(k)+1\right) \quad \text { for } k \in[0, T+1],
$$

where $\phi_{1}$ is defined in Lemma 2.2.

It is worth remarking here that an estimate for $\lambda_{0}$ will be given in the proof of Lemma 2.11 .

We first give some lemmas which will help us to prove Theorem 2.1.

Lemma 2.2 [1]. Consider the following eigenvalue problem:

$$
\begin{gathered}
-\Delta^{2} u(k-1)=\lambda u(k), \quad k \in[1, T], \\
u(0)=u(T+1)=0 .
\end{gathered}
$$

Then the eigenvalues are

$$
\lambda_{m}=4 \sin ^{2} \frac{m \pi}{2(T+1)}, \quad 1 \leq m \leq T,
$$

and the corresponding eigenfunctions are

$$
\phi_{m}(k)=\sin \frac{m k \pi}{T+1} \quad \text { for } k \in[0, T+1], 1 \leq m \leq T .
$$

Lemma 2.3 [3]. Let $G_{a}(k, l)$ be Green's function of the BVP

$$
\begin{gathered}
-\Delta^{2} u(k-1)+a(t) u(t)=0 \quad \text { for } t \in[1, T], \\
u(0)=0, \quad u(T+1)=0 .
\end{gathered}
$$


Haishen Lü et al. 3

Then

$$
0<G_{a}(k, l) \leq G_{a}(l, l) \quad \text { for every }(k, l) \in[1, T] \times[1, T],
$$

where $a \in C[1, T]$ and $a(k) \geq 0$ for $k \in[1, T]$.

Remark 2.4. If $a(k) \equiv 0$ for $k \in[1, T]$, then

$$
G_{0}(k, l)=\frac{1}{T+1}\left\{\begin{array}{ll}
l(T+1-k), & l \in[0, k-1], \\
k(T+1-l), & l \in[k, T+1],
\end{array} \quad \text { for } k \in[1, T] .\right.
$$

Next we consider the boundary value problem

$$
\begin{gathered}
-\Delta^{2} u(k-1)+a(k) u(k)=f(k), \quad k \in[1, T], \\
u(0)=0=u(T+1),
\end{gathered}
$$

where $a, f \in C[1, T]$ and $a(k) \geq 0$ for $k \in[1, T]$.

Let $A: C[1, T] \rightarrow C[1, T]$ be the operator defined by

$$
A u(k):=\sum_{l=1}^{T} G_{a}(k, l) u(l) .
$$

It is easy to see that $A$ is a completely continuous operator (see [3]).

Note that if $u \in C[0, T+1], u(0)=u(T+1)=0$, and

$$
u(k)=A(f)(k) \text { for } k \in[1, T],
$$

then $u$ is a solution of (2.10).

From Lemma 2.3, we have the following lemma.

LeMma 2.5. The following statements hold:

(i) for any $f \in C[1, T],(2.10)$ is uniquely solvable and $u=A(f)$;

(ii) if $f(k) \geq 0$ for $k \in[1, T]$, then the solution of (2.10) is nonnegative.

Corollary 2.6. If $f_{1}(k) \leq f_{2}(k)$ for $k \in[1, T]$, then $A\left(f_{1}\right)(k) \leq A\left(f_{2}\right)(k)$ for $k \in[1, T]$.

Lemma 2.7. Suppose $(G)$ and $(H)$ hold. Let $n_{0} \in \mathbb{N}$. Assume that for every $n>n_{0}$, there exist $a_{n} \in C[1, T], 0 \leq a_{n}$, and there exist $\bar{u}, \bar{u}_{n}, \hat{u}_{n}, \hat{u} \in C[0, T+1]$ such that

$$
0<\bar{u}(k) \leq \bar{u}_{n}(k) \leq \hat{u}_{n}(k) \leq \hat{u}(k) \quad \text { for } k \in[1, T]
$$

and $\hat{u}(0)=\hat{u}(T+1)=0$. If

$$
\begin{aligned}
& -\Delta^{2} \bar{u}_{n}(k-1)+a_{n}(k) \bar{u}_{n}(k) \\
& \quad \leq g\left(k, \frac{1}{n}+v(k)\right)+\lambda h(k, v(k))+a_{n}(k) v(k) \quad \text { for } k \in[1, T], \\
& -\Delta^{2} \hat{u}_{n}(k-1)+a_{n}(k) \hat{u}_{n}(k) \\
& \quad \geq g\left(k, \frac{1}{n}+v(k)\right)+\lambda h(k, v(k))+a_{n}(k) v(k) \quad \text { for } k \in[1, T],
\end{aligned}
$$




\section{Singular discrete BVPs}

where $\lambda \geq 0$ and $v \in\left[\bar{u}_{n}, \hat{u}_{n}\right]=\left\{u \in C[0, T+1], \bar{u}_{n}(k) \leq u(k) \leq \hat{u}_{n}(k)\right.$ for $\left.k \in[0, T+1]\right\}$, then problem (1.1) has a solution $u \in C[0, T+1]$ such that $\bar{u}(k) \leq u(k) \leq \hat{u}(k)$ for $k \in$ $[0, T+1]$.

Proof. Fix $v \in[\bar{u}, \hat{u}]$. From Lemma 2.5, there exists $\Psi(v) \in C[0, T+1]$ such that

$$
\begin{gathered}
-\Delta^{2} \Psi(v)(k-1)+a_{n}(k) \Psi(v)(k) \\
=g\left(k, \frac{1}{n}+v(k)\right)+\lambda h(k, v(k))+a_{n}(k) v(k) \quad \text { for } k \in[1, T], \\
\Psi(v)(0)=\Psi(v)(T+1)=0 .
\end{gathered}
$$

Then

$$
\Psi(v)(k)=A\left(g\left(\cdot, \frac{1}{n}+v\right)+\lambda h(\cdot, v)+a_{n} v\right)(k) \quad \text { for } k \in[1, T]
$$

Note also that $\Psi: C[0, T+1] \rightarrow C[0, T+1]$ is a completely continuous operator. By Corollary 2.6, we have

$$
\bar{u}_{n}(k) \leq \Psi(v)(k) \leq \hat{u}_{n}(k) \text { for } k \in[0, T+1] .
$$

From Schauder's fixed point theorem (note that $\Psi z:[\bar{u}, \hat{u}] \rightarrow[\bar{u}, \hat{u}]$ ), there exists $u_{n} \in$ $C[0, T+1]$ such that $\bar{u}_{n}(k) \leq u_{n}(k) \leq \hat{u}_{n}(k)$ and $\Psi\left(u_{n}\right)(k)=u_{n}(k)$ for $k \in[1, T]$. Note that

$$
\begin{gathered}
-\Delta^{2} u_{n}(k-1)=g\left(k, \frac{1}{n}+u_{n}(k)\right)+\lambda h\left(k, u_{n}(k)\right) \quad \text { for } k \in[1, T], \\
u_{n}(0)=u_{n}(T+1)=0 .
\end{gathered}
$$

Let $m:=\min \{\bar{u}(k): k \in[1, T]\}>0$ and $M:=\max \{\hat{u}(k): k \in[1, T]\}$. Then

$$
m \leq u_{n}(k) \leq M \quad \text { for } k \in[1, T], n=1,2, \ldots,
$$

and for $k \in[1, T]$, we have

$$
\left|g\left(k, \frac{1}{n}+u_{n}(k)\right)+\lambda h\left(k, u_{n}(k)\right)\right| \leq g_{2}(k, m)+\lambda h_{2}(k, M)
$$

From the Arzela-Ascoli theorem, there exist a $u \in C[0, T+1]$ and a subsequence $\left\{u_{n_{m}}\right\}_{m \in \mathbb{N}}$ converging to $u$ in $C[0, T+1]$, and of course

$$
u(k)=\lim _{m \rightarrow \infty} u_{n_{m}}(k) \quad \text { for } k \in[0, T+1]
$$


Observe that $u_{n_{m}} \in[\bar{u}, \hat{u}]$, so $u(0)=u(T+1)=0$ and $u \in C[0, T+1]$ with $u>0$ in $[1, T]$. Also,

$$
\begin{aligned}
u(k) & =\lim _{m \rightarrow \infty} \sum_{l=1}^{T} G_{0}(k, l)\left[g\left(l, \frac{1}{n}+u_{n_{m}}(l)\right)+\lambda h\left(l, u_{n_{m}}(l)\right)\right] \\
& =\sum_{l=1}^{T} G_{0}(k, l)[g(l, u(l))+\lambda h(l, u(l))] .
\end{aligned}
$$

As a result

$$
\begin{gathered}
-\Delta^{2} u(k-1)=g(k, u(k))+\lambda h(k, u(k)) \quad \text { for } k \in[1, T], \\
u(0)=u(T+1)=0 .
\end{gathered}
$$

Lemma 2.8. Let $\psi:[1, T] \times(0, \infty) \rightarrow(0, \infty)$ be a continuous function with $\psi(k, \cdot)$ strictly decreasing. Then the problem

$$
\begin{gathered}
-\Delta^{2} \omega(k-1)=\psi\left(k, \omega+\frac{1}{n}\right) \quad \text { for } k \in[0, T], \\
\omega(0)=\omega(T+1)=0
\end{gathered}
$$

has a solution $\omega_{n} \in C[0, T+1]$ such that

$$
\omega_{n}(k) \leq \omega_{n+1}(k) \leq 1+\omega_{1}(k) \quad \text { for } k \in[0, T+1], n \in \mathbb{N} .
$$

If $\omega(k)=\lim _{n \rightarrow \infty} \omega_{n}(k)$ for $k \in[0, T+1]$, then

$$
\begin{gathered}
\omega \in C[0, T+1], \quad \omega(k)>0, \quad \text { for } k \in[1, T], \\
-\Delta^{2} \omega(k-1)=\psi(k, \omega) \quad \text { for } k \in[1, T], \\
\omega(0)=\omega(T+1)=0 .
\end{gathered}
$$

Proof. There exists $\chi_{1} \in C[0, T+1]$ such that

$$
\begin{gathered}
-\Delta^{2} \chi_{1}(k-1)=\psi(k, 1), \\
\chi_{1}(0)=\chi_{1}(T+1)=0, \\
\chi_{1}(k)>0 \quad \text { for } k \in[1, T] .
\end{gathered}
$$

Notice that

$$
\begin{gathered}
-\Delta^{2} \chi_{1}(k-1)=\psi(k, 1) \geq \psi\left(k, 1+\chi_{1}(k)\right), \\
0 \leq \psi(k, 1+0) .
\end{gathered}
$$

By a standard upper-lower solution method [2, page 264], there exists $\omega_{1} \in C[0, T+1]$ such that

$$
\begin{gathered}
-\Delta^{2} \omega_{1}(k-1)=\psi\left(k, 1+\omega_{1}(k)\right) \quad \text { for } k \in[1, T], \\
\omega_{1}(0)=\omega_{1}(T+1)=0 .
\end{gathered}
$$


6 Singular discrete BVPs

Suppose that there exists $\omega_{n} \in C[0, T+1]$ such that

$$
\begin{gathered}
-\Delta^{2} \omega_{n}(k-1)=\psi\left(k, \frac{1}{n}+\omega_{n}(k)\right), \\
\omega_{n}(0)=\omega_{n}(T+1)=0, \\
\omega_{n}(k)>0 \quad \text { for } k \in[1, T] .
\end{gathered}
$$

We know that there exist $\chi_{n+1} \in C[0, T+1]$ such that

$$
\begin{gathered}
-\Delta^{2} \chi_{n+1}(k-1)=\psi\left(k, \frac{1}{n+1}\right), \\
\chi_{n+1}(0)=\chi_{n+1}(T+1)=0, \\
\chi_{n+1}(k)>0 \quad \text { for } k \in[1, T] .
\end{gathered}
$$

Then

$$
\begin{gathered}
-\Delta^{2} \chi_{n+1}(k-1)=\psi\left(k, \frac{1}{n+1}\right) \geq \psi\left(k, \frac{1}{n+1}+\chi_{n+1}(k)\right), \\
-\Delta^{2} \omega_{n}(k-1)=\psi\left(k, \frac{1}{n}+\omega_{n}(k)\right) \leq \psi\left(k, \frac{1}{n+1}+\omega_{n}(k)\right) \quad \text { for } k \in[1, T], \\
\omega_{n}(0)=\omega_{n}(T+1)=0, \\
\omega_{n}(k)=\sum_{l=1}^{T} G_{0}(k, l) \psi\left(l, \frac{1}{n}+\omega_{n}(l)\right) \leq \sum_{l=1}^{T} G_{0}(k, l) \psi\left(l, \frac{1}{n+1}\right)=\chi_{n+1}(k) \quad \text { for } k \in[1, T] .
\end{gathered}
$$

By a standard upper-lower solution method, there exist $\omega_{n+1} \in C[0, T+1]$ such that

$$
\begin{gathered}
-\Delta^{2} \omega_{n+1}(k-1)=\psi\left(k, \frac{1}{n+1}+\omega_{n+1}\right) \quad \text { for } k \in[1, T], \\
\omega_{n+1}(0)=\omega_{n+1}(T+1)=0 \\
\omega_{n}(k) \leq \omega_{n+1}(k) \quad \text { for } k \in[0, T+1] .
\end{gathered}
$$

Next we prove

$$
\omega_{n+1}(k)+\frac{1}{n+1} \leq \omega_{n}(k)+\frac{1}{n} \quad \text { for } k \in[0, T+1] .
$$

To see this, we consider the problem

$$
\begin{aligned}
-\Delta^{2} v(k-1) & =\psi(k, v) \quad \text { for } k \in[1, T], \\
v(0) & =v(T+1)=\frac{1}{n} .
\end{aligned}
$$

Then $v_{n}(k)=1 / n+\omega_{n}(k)$ for $k \in[0, T+1]$ is a solution of $(2.36)_{n}$. We next prove

$$
v_{n+1}(k) \leq v_{n}(k) \text { for } k \in[0, T+1]
$$


Since $v_{n+1}(0)=1 /(n+1)<1 / n=v_{n}(0), v_{n+1}(1)=1 /(n+1)<1 / n=v_{n}(1)$, we need only to prove that

$$
v_{n+1}(k) \leq v_{n}(k) \text { for } k \in[1, T] .
$$

If this is not true, then there exist $m \in[1, T]$ with $v_{n+1}(m)>v_{n}(m)>0$. Let $\sigma$ be the point where $v_{n+1}(k)-v_{n}(k)$ assumes its maximum over $[1, T]$. Certainly, $v_{n+1}(\sigma)-v_{n}(\sigma)>0$. Let $y(k)=v_{n+1}(k)-v_{n}(k)$. Now $y(\sigma) \geq y(\sigma+1)$ and $y(\sigma) \geq y(\sigma-1)$ imply that

$$
2 y(\sigma) \geq y(\sigma+1)+y(\sigma-1)
$$

that is,

$$
y(\sigma+1)+y(\sigma-1)-2 y(\sigma) \leq 0 .
$$

Thus

$$
\Delta^{2} y(\sigma-1) \leq 0
$$

On the other hand, since $v_{n+1}(\sigma)>v_{n}(\sigma)$, we have

$$
\begin{aligned}
\Delta^{2} y(\sigma-1) & =\Delta^{2} v_{n+1}(\sigma-1)-\Delta^{2} v_{n}(\sigma-1) \\
& =-\psi\left(\sigma, v_{n+1}(\sigma)\right)+\psi\left(\sigma, v_{n}(\sigma)\right) \\
& =\psi\left(\sigma, v_{n}(\sigma)\right)-\psi\left(\sigma, v_{n+1}(\sigma)\right)>0 .
\end{aligned}
$$

This is a contradiction. Thus $v_{n+1}(k) \leq v_{n}(k)$ for $k \in[1, T]$, and so

$$
0<\frac{1}{n+1}+\omega_{n+1} \leq \omega_{n}+\frac{1}{n}
$$

Also notice that

$$
\omega_{1}(k) \leq \omega_{n}(k) \leq \omega_{n+1}(k) \leq 1+\omega_{1}(k) \text { for } k \in[0, T+1], n \in \mathbb{N} .
$$

Now with

$$
\omega(k)=\lim _{n \rightarrow \infty} \omega_{n}(k)=\sup _{n \in \mathbb{N}} \omega_{n}(k) \quad \text { for } k \in[0, T+1],
$$

we have

$$
\begin{aligned}
0<\omega_{1}(k) \leq \omega(k) & \leq 1+\omega_{1}(k) \quad \text { for } k \in[1, T], \\
\omega(0) & =\omega(T+1)=0 .
\end{aligned}
$$


8 Singular discrete BVPs

Also for $k \in[1, T]$, we have

$$
\begin{aligned}
\omega(k) & =\lim _{n \rightarrow \infty} \omega_{n}(k) \\
& =\lim _{n \rightarrow \infty} \sum_{l=1}^{T} G(k, l) \psi\left(l, \frac{1}{n}+\omega_{n}(l)\right) \\
& =\sum_{l=1}^{T} G(k, l) \psi(l, \omega(l)),
\end{aligned}
$$

so

$$
\begin{aligned}
-\Delta^{2} \omega(k-1) & =\psi(k, \omega) \quad \text { for } k \in[0, T], \\
\omega(0) & =\omega(T+1)=0 .
\end{aligned}
$$

Lemma 2.9. Suppose that $m:[1, T] \times[0, \infty) \rightarrow[0, \infty)$ is a continuous function such that

$$
\begin{gathered}
m(k, \cdot) \text { is increasing, } \\
\lim _{u \rightarrow+\infty} \frac{m(k, u)}{u}=0 \quad \text { for } k \in[1, T] .
\end{gathered}
$$

There exist $R_{0}>0$ and $\tilde{v} \in C[0, T+1]$ with $0 \leq \tilde{v} \leq R_{0} \phi_{1}$ and

$$
\begin{aligned}
-\Delta^{2} \widetilde{v}(k-1) & =m(k, \widetilde{v}) \quad \text { for } k \in[1, T], \\
\widetilde{v}(0) & =\widetilde{v}(T+1)=0 .
\end{aligned}
$$

Proof. We first prove that

$$
\lim _{\mathbb{R} \rightarrow \infty} \frac{\sum_{l=1}^{T} G_{0}(k, l) m(l, v(l))}{R \phi_{1}(k)}=0 \quad \text { for } k \in[1, T],
$$

for all $v \in C[0, T+1]$ with $0 \leq v(i) \leq R \phi_{1}(i)$ for $i \in[0, T+1]$.

From (2.49), for all $\sigma>0$, there exist $s_{\sigma}>0$ such that

$$
m(k, s) \leq \sigma s \quad \text { for } k \in[1, T] \text { and } s_{\sigma} \leq s .
$$

As a result,

$$
\left.m(k, v(k))\right|_{0 \leq v(k) \leq R \phi_{1}(k)} \leq m\left(k, s_{\sigma}\right)+\sigma v(k) \leq m\left(k, s_{\sigma}\right)+\sigma R \phi_{1}(k) \quad \text { for } k \in[1, T],
$$

so

$$
\begin{aligned}
\frac{1}{\phi_{1}(k)} \sum_{l=1}^{T} G_{0}(k, l) m(l, v(l)) & \leq \frac{1}{\phi_{1}(k)}\left[\sum_{l=1}^{T} G_{0}(k, l) m\left(l, s_{\sigma}\right)+R \sigma \sum_{l=1}^{T} G_{0}(k, l) \phi_{1}(l)\right] \\
& =\frac{1}{\phi_{1}(k)} \sum_{l=1}^{T} G_{0}(k, l) m\left(l, s_{\sigma}\right)+\frac{R \sigma}{\lambda_{1}},
\end{aligned}
$$


Haishen Lü et al. 9

and consequently

$$
\frac{1}{R \phi_{1}(k)} \sum_{l=1}^{T} G_{0}(k, l) m(l, v(l)) \leq \frac{1}{R \phi_{1}(k)} \sum_{l=1}^{T} G_{0}(k, l) m\left(l, s_{\sigma}\right)+\frac{\sigma}{\lambda_{1}},
$$

so (2.51) follows. Thus there exist $R_{0}>0$ such that if $v \in C[0, T+1]$ and $0 \leq v(i) \leq$ $R_{0} \phi_{1}(i)$ for $i \in[0, T+1]$, then

$$
\frac{1}{R_{0} \phi_{1}(k)} \sum_{l=1}^{T} G_{0}(k, l) m(l, v(l)) \leq 1 \quad \text { for } k \in[1, T]
$$

and so

$$
0 \leq \sum_{l=1}^{T} G_{0}(k, l) m(l, v(l)) \leq R_{0} \phi_{1}(k) \quad \text { for } k \in[1, T] .
$$

Let $\Phi: C[1, T] \rightarrow C[1, T]$ be the operator defined by

$$
(\Phi v)(k):=\sum_{l=1}^{T} G_{0}(k, l) m(l, v(l)) \quad \text { for } v \in C[1, T], k \in[1, T] .
$$

It is easy to see that $\Phi$ is a completely continuous operator. Also if $v \in C[0, T+1]$ and $0 \leq$ $v(k) \leq R_{0} \phi_{1}(k)$ for $k \in[1, T]$, then $0 \leq \Phi(v)(k) \leq R_{0} \phi_{1}(k)$ for $k \in[1, T]$, so Schauder's fixed point theorem guarantees that there exists $\tilde{v} \in\left[0, R_{0} \phi_{1}\right]$ with $\Phi(\tilde{v})=\tilde{v}$, that is,

$$
-\Delta^{2} \widetilde{v}(k-1)=m(k, \tilde{v}), \quad \tilde{v}(0)=\tilde{v}(T+1)=0 .
$$

Corollary 2.10. Let $\psi(k, s), m(k, s),\left(\omega_{n}\right)_{n \in \mathbb{N}}$, and $R_{0}>0$ be as in Lemmas 2.8 and 2.9. Then there exist $\left\{\tilde{v}_{n}\right\}_{n \in \mathbb{N}} \subset C[0, T+1]$ and $0 \leq \widetilde{v}_{n} \leq R_{0} \phi_{1}$ such that

$$
\begin{gathered}
-\Delta^{2} \tilde{v}_{n}(k-1)=m\left(k, \omega_{n}+\tilde{v}_{n}\right) \quad \text { for } k \in[1, T], \\
\tilde{v}_{n}(0)=\widetilde{v}_{n}(T+1)=0, \\
-\Delta^{2}\left(w_{n}+\widetilde{v}_{n}\right)(k-1) \geq \psi\left(k, \frac{1}{n}+\omega_{n}+\widetilde{v}_{n}\right)+m\left(k, \omega_{n}+\widetilde{v}_{n}\right) \quad \text { for } k \in[1, T] .
\end{gathered}
$$

Proof. Let $n \in \mathbb{N}$ be fixed. Then $m\left(k, \omega_{n}+s\right)$ satisfies the conditions of Lemma 2.9, so there exists $\widetilde{v}_{n} \in C[0, T+1]$ with $0 \leq \widetilde{v}_{n} \leq R_{0} \phi_{1}$ such that $(2.60)$ holds and

$$
\begin{aligned}
-\Delta^{2}\left(w_{n}+\tilde{v}_{n}\right)(k-1) & =-\Delta^{2} w_{n}(k-1)-\Delta^{2} \widetilde{v}_{n}(k-1)=\psi\left(k, \frac{1}{n}+\omega_{n}\right)+m\left(k, \omega_{n}+\widetilde{v}_{n}\right) \\
& \geq \psi\left(k, \frac{1}{n}+\omega_{n}+\widetilde{v}_{n}\right)+m\left(k, \omega_{n}+\widetilde{v}_{n}\right) \quad \text { for } k \in[1, T] .
\end{aligned}
$$


Lemma 2.11. Suppose $(G)$ and $(H)$ hold. Then there exist $\lambda_{0} \geq 0, c>0$ such that for all $\lambda \geq \lambda_{0}$, there exist $R_{c}>c, \bar{u} \in C([0, T+1])$ with $c \phi_{1}(k) \leq \bar{u}(k) \leq R_{c} \phi_{1}(k)$ and

$$
\begin{aligned}
-\Delta^{2} \bar{u}(k-1)= & -g_{1}(k, \bar{u})+\lambda h_{1}(k, \bar{u}) \quad \text { for } k \in[1, T], \\
& \bar{u}(0)=\bar{u}(T+1)=0 .
\end{aligned}
$$

Proof. Let us consider the operator $T_{\lambda}: C[1, T] \rightarrow C[1, T]$ given by

$$
T_{\lambda}(v)(k):=\frac{1}{\phi_{1}(k)} \sum_{l=1}^{T} G_{0}(k, l)\left[-g_{1}\left(l, v(l) \phi_{1}(l)\right)+\lambda h_{1}\left(l, v(l) \phi_{1}(l)\right)\right] \quad \text { for } k \in[1, T] .
$$

By $(\mathrm{H})$, there exists $\bar{s} \geq 0$ such that $0<h_{1}(k, \bar{s})$ for $k \in[1, T]$. We let

$$
c=2 \frac{\bar{s}+1}{\left|\phi_{1}\right|_{\infty}}, \quad \Theta=\left\{k \in[1, T]: \frac{\left|\phi_{1}\right|_{\infty}}{2}<\phi_{1}(k)\right\} .
$$

Note that $\Theta$ is nonempty. If $k \in \Theta, v \in C[0, T+1]$, and $c \leq v$, we have

$$
\bar{s}=\frac{c\left|\phi_{1}\right|_{\infty}}{2}-1 \leq \frac{c\left|\phi_{1}\right|_{\infty}}{2} \leq c \phi_{1}(k) \leq v(k) \phi_{1}(k)
$$

so

$$
h_{1}(k, \bar{s}) \leq h_{1}\left(k, v(k) \phi_{1}(k)\right)
$$

for all $v \in C[0, T+1]$ with $c \leq v$. Let

$$
\rho=\min _{k \in[1, T]} \frac{1}{\phi_{1}(k)} \sum_{l \in \Theta} G_{0}(k, l) h_{1}(l, \bar{s})>0,
$$

and note for $v \in C[0, T+1]$ with $c \leq v$ that

$$
\begin{aligned}
\frac{1}{\phi_{1}(k)} \sum_{l=1}^{T} G_{0}(k, l) h_{1}\left(l, v(l) \phi_{1}(l)\right) & \geq \frac{1}{\phi_{1}(k)} \sum_{l \in \Theta} G_{0}(k, l) h_{1}\left(l, v(l) \phi_{1}(l)\right) \\
& \geq \frac{1}{\phi_{1}(k)} \sum_{l \in \Theta} G_{0}(k, l) h_{1}(l, \bar{s}) \quad(\text { see }(2.66)) \\
& \geq \min _{k \in[1, T]} \frac{1}{\phi_{1}(k)} \sum_{l \in \Theta} G_{0}(k, l) h_{1}(l, \bar{s}) \\
& =\rho \quad \forall k \in[1, T]
\end{aligned}
$$

that is,

$$
\frac{\phi_{1}(k)}{\sum_{l=1}^{T} G_{0}(k, l) h_{1}\left(l, v(l) \phi_{1}(l)\right)} \leq \frac{1}{\rho}
$$


On the other hand, for all $v \in C[0, T+1]$ with $v \geq c$, we have

$$
\begin{aligned}
& c+\frac{1}{\phi_{1}(k)} \sum_{l=1}^{T} G_{0}(k, l) g_{1}\left(l, v(l) \phi_{1}(l)\right) \\
& \quad \leq c+\frac{1}{\phi_{1}(k)} \sum_{l=1}^{T} G_{0}(k, l) g_{1}\left(l, c \phi_{1}(l)\right) \leq c+\frac{1}{\phi_{1}(k)} \sum_{l=1}^{T} G_{0}(k, l) g_{1}(l, c \mu),
\end{aligned}
$$

where $\mu=\min _{1 \leq l \leq T} \phi_{1}(l)$. Thus, for all $v \in C[0, T+1]$ with $v(k) \geq c$, we have

$$
\begin{aligned}
& \frac{c+\left(1 / \phi_{1}(k)\right) \sum_{l=1}^{T} G_{0}(k, l) g_{1}\left(l, v(l) \phi_{1}(l)\right)}{\left(\sum_{l=1}^{T} G_{0}(k, l) h_{1}\left(l, v(l) \phi_{1}(l)\right)\right) / \phi_{1}(k)} \\
& \leq \frac{1}{\rho}\left(c+\frac{1}{\phi_{1}(k)} \sum_{l=1}^{T} G_{0}(k, l) g_{1}(l, c \mu)\right) \quad \text { for } k \in[1, T] .
\end{aligned}
$$

Let

$$
\lambda_{0}:=\sup \left\{\left|\frac{c+\left(1 / \phi_{1}(k)\right) \sum_{l=1}^{T} G_{0}(k, l) g_{1}\left(l, v(l) \phi_{1}(l)\right)}{\left(\sum_{l=1}^{T} G_{0}(k, l) h_{1}\left(l, v(l) \phi_{1}(l)\right)\right) / \phi_{1}(k)}\right|_{*}: v \in C[0, T+1], c \leq v\right\}<\infty,
$$

where $|u|_{*}=\max [1, T]|u(k)|$. Then, for all $\lambda \geq \lambda_{0}, v \in C[0, T+1]$, and $c \leq v$, we have for $k \in[1, T]$ that

$$
\frac{c+\left(1 / \phi_{1}(k)\right) \sum_{l=1}^{T} G_{0}(k, l) g_{1}\left(l, v(l) \phi_{1}(l)\right)}{\left(\sum_{l=1}^{T} G_{0}(k, l) h_{1}\left(l, v(l) \phi_{1}(l)\right)\right) / \phi_{1}(k)} \leq \lambda,
$$

that is,

$$
c+\frac{1}{\phi_{1}(k)} \sum_{l=1}^{T} G_{0}(k, l) g_{1}\left(l, v(l) \phi_{1}(l)\right) \leq \frac{\lambda}{\phi_{1}(k)} \sum_{l=1}^{T} G_{0}(k, l) h_{1}\left(l, v(l) \phi_{1}(l)\right),
$$

so

$$
\begin{aligned}
c & \leq \frac{1}{\phi_{1}(k)} \sum_{l=1}^{T} G_{0}(k, l)\left(-g_{1}\left(l, v(l) \phi_{1}(l)\right)+\lambda h_{1}\left(l, v(l) \phi_{1}(l)\right)\right) \\
& =T_{\lambda}(v)(k) \quad \text { for } k \in[1, T] .
\end{aligned}
$$

On the other hand, for all $v \in C[0, T+1]$ with $v \geq c$, we have

$$
\begin{aligned}
\frac{1}{\phi_{1}(k)} \sum_{l=1}^{T} G_{0}(k, l) g_{1}\left(l, v(l) \phi_{1}(l)\right) & \leq \frac{1}{\phi_{1}(k)} \sum_{l=1}^{T} G_{0}(k, l) g_{1}\left(l, c \phi_{1}(l)\right) \\
& \leq \max _{k \in[1, T]} \frac{1}{\phi_{1}(k)} \sum_{l=1}^{T} G_{0}(k, l) g_{1}\left(l, c \phi_{1}(l)\right),
\end{aligned}
$$


so

$$
\lim _{\mathbb{R} \rightarrow \infty} \frac{1}{R}\left[\frac{1}{\phi_{1}(k)} \sum_{l=1}^{T} G_{0}(k, l) g_{1}\left(l, v(l) \phi_{1}(l)\right)\right]=0,
$$

for all $v \in C[0, T+1]$ with $v \geq c$ and $k \in[1, T]$. Essentially the same reasoning as in the proof of $(2.51)$ yields (note that $\lim _{u \rightarrow \infty}\left(h_{1}(k, u) / u\right)=0$ for $k \in[1, T]$ )

$$
\lim _{\mathbb{R} \rightarrow \infty} \frac{1}{R}\left[\frac{1}{\phi_{1}(k)} \sum_{l=1}^{T} G_{0}(k, l) h_{1}\left(l, v(l) \phi_{1}(l)\right)\right]=0
$$

for all $v \in C[0, T+1]$ with $0 \leq v(i) \leq R$ and $i \in[1, T]$. Thus if $\lambda \geq \lambda_{0}$, there exists $R_{c}>c$ with $T_{\lambda}\left(\left[c, R_{c}\right]\right) \subset\left[c, R_{c}\right]$.

It is easy to see that $T_{\lambda}$ is a completely continuous operator, so Schauder's fixed point theorem guarantees that there exists $\bar{v} \in\left[c, R_{c}\right]$ with $T_{\lambda}(\bar{v})=\bar{v}$, that is,

$$
\bar{v}(k) \phi_{1}(k)=\sum_{l=1}^{T} G_{0}(k, l)\left(-g_{1}\left(l, \bar{v}(l) \phi_{1}(l)\right)+\lambda h_{1}\left(l, \bar{v}(l) \phi_{1}(l)\right)\right) .
$$

The function $\bar{u}=\phi_{1} \bar{v}$ satisfies (2.62).

Proof of Theorem 2.1. Let $\lambda_{0}>0, c>0$, and $\bar{u} \in(C[0, T+1])$ be defined as in Lemma 2.11. Also let

$$
\begin{gathered}
\psi(k, s)=g_{2}(k, s)+\lambda h_{1}(k, \bar{u}(k)) \quad \text { for } k \in[1, T], \\
m(k, s)=\lambda h_{2}(k, s),
\end{gathered}
$$

where $\lambda \geq \lambda_{0}$.

From $(\mathrm{G})$, we notice that $\psi$ satisfies the assumptions of Lemma 2.8. As a result, there exist $\omega, \omega_{n} \in C[0, T+1]$ such that

$$
\begin{gathered}
-\Delta^{2} \omega_{n}(k-1)=g_{2}\left(k, \frac{1}{n}+\omega_{n}\right)+\lambda h_{1}(k, \bar{u}(k)) \quad \text { for } k \in[1, T], \\
\omega_{n}(0)=\omega_{n}(T+1)=0, \\
\omega(k)=\lim _{n \rightarrow \infty} \omega_{n}(k) \quad \text { for } k \in[0, T+1] .
\end{gathered}
$$

From $(\mathrm{H})$, we notice that $m$ satisfies the assumptions of Lemma 2.9. As a result from Corollary 2.10, there exist $R_{0}>0$ and $\tilde{v}_{n} \in C([0, T+1]), 0 \leq \widetilde{v}_{n}(k) \leq R_{0} \phi_{1}(k)$ for $k \in$ $[0, T+1]$ such that

$$
\begin{gathered}
-\Delta^{2} \widetilde{v}_{n}(k-1)=\lambda h_{2}\left(k, \omega_{n}+\widetilde{v}_{n}\right) \quad \text { for } k \in[1, T], \\
\widetilde{v}_{n}(0)=\widetilde{v}_{n}(T+1)=0, \\
-\Delta^{2}\left(\omega_{n}+\widetilde{v}_{n}\right)(k-1) \geq g_{2}\left(k, \frac{1}{n}+\omega_{n}+\widetilde{v}_{n}\right)+\lambda h_{1}(k, \bar{u}(k))+\lambda h_{2}\left(k, \omega_{n}+\widetilde{v}_{n}\right) \quad \text { for } k \in[1, T] .
\end{gathered}
$$


Let

$$
\widehat{u}_{n}(k)=\omega_{n}(k)+\widetilde{v}_{n}(k) \quad \text { for } k \in[0, T+1] .
$$

Then, $\hat{u}_{n} \in C[0, T+1], \hat{u}_{n}(1)=\hat{u}_{n}(T+1)=0$.

We let

$$
\hat{u}(k)=\omega(k)+R_{0} \phi_{1}(k) \quad \text { for } k \in[0, T+1],
$$

so

$$
0 \leq \widehat{u}_{n}(k) \leq \widehat{u}(k) \quad \text { for } k \in[0, T+1] .
$$

From Lemma 2.11, we have

$$
\begin{aligned}
-\Delta^{2} \bar{u}(k-1) & =-g_{1}(k, \bar{u}(k))+\lambda h_{1}(k, \bar{u}(k)) \\
& \leq \lambda h_{1}(k, \bar{u}(k)) \\
& \leq \lambda h_{1}(k, \bar{u}(k))+g_{2}\left(k, \frac{1}{n}+\hat{u}_{n}(k)\right)+\lambda h_{2}\left(k, \hat{u}_{n}(k)\right) \\
& \leq-\Delta^{2} \hat{u}_{n}(k-1) \quad \text { for } k \in[1, T],
\end{aligned}
$$

that is,

$$
-\Delta^{2}\left(\bar{u}-\hat{u}_{n}\right)(k-1) \leq 0 .
$$

A standard argument (see the argument to show (2.35)) yields

$$
\bar{u}(k) \leq \hat{u}_{n}(k) \text { for } k \in[1, T] .
$$

Let

$$
a_{n}(k)=\sup \left\{\left|\frac{\partial}{\partial s} g_{2}\left(k, \frac{1}{n}+s\right)\right|: 0<s\right\},
$$

and notice that $s \rightarrow g_{2}(k, 1 / n+s)+a(k) s$ is increasing. Let $\bar{u}_{n}=\bar{u}$. From (2.85) and (2.88), we have

$$
\bar{u}(k)=\bar{u}_{n}(k) \leq \hat{u}_{n}(k) \leq \widehat{u}(k) \text { for } k \in[0, T+1] .
$$

Also for $v \in C[1, T]$ with $\bar{u}_{n}(k) \leq v(k) \leq \hat{u}_{n}(k), k \in[1, T]$, we have

$$
\begin{aligned}
-\Delta^{2} \bar{u}_{n}(k-1)+a_{n}(k) \bar{u}_{n}(k) & =-g_{1}\left(k, \bar{u}_{n}(k)\right)+\lambda h_{1}\left(k, \bar{u}_{n}(k)\right)+a_{n}(k) \bar{u}_{n}(k) \\
& \leq-g_{1}(k, v(k))+\lambda h_{1}(k, v(k))+a_{n}(k) v(k) \\
& \leq-g_{1}\left(k, \frac{1}{n}+v(k)\right)+\lambda h_{1}(k, v(k))+a_{n}(k) v(k) \\
& \leq g\left(k, \frac{1}{n}+v(k)\right)+\lambda h(k, v)+a_{n}(k) v(k) \text { for } k \in[1, T],
\end{aligned}
$$

so (2.14) holds. 


\section{Singular discrete BVPs}

Also for $v \in C[1, T]$ with $\bar{u}_{n}(k) \leq v(k) \leq \hat{u}_{n}(k), k \in[1, T]$, we have

$$
\begin{aligned}
-\Delta^{2} \hat{u}_{n}(k & -1)+a_{n}(k) \hat{u}_{n}(k) \\
& \geq g_{2}\left(k, \frac{1}{n}+\hat{u}_{n}(k)\right)+\lambda h_{1}(k, \bar{u}(k))+\lambda h_{2}\left(k, \hat{u}_{n}(k)\right)+a_{n}(k) \hat{u}_{n}(k) \\
& \geq g_{2}\left(k, \frac{1}{n}+\hat{u}_{n}(k)\right)+a_{n}(k) \hat{u}_{n}(k)+\lambda h_{2}\left(k, \hat{u}_{n}(k)\right) \\
& \geq g_{2}\left(k, \frac{1}{n}+v(k)\right)+a_{n}(k) v(k)+\lambda h_{2}(k, v(k)) \\
& \geq g\left(k, \frac{1}{n}+v(k)\right)+\lambda h(k, v(k))+a_{n}(k) v(k) \quad \text { for } k \in[1, T],
\end{aligned}
$$

so (2.15) holds. Lemma 2.7 guarantees that there exists a solution $u \in C[0, T+1]$ to $(1.1)$ with

$$
\bar{u}(k) \leq u(k) \leq \widehat{u}(k) \quad \text { for } k \in[0, T+1] .
$$

Moreover, because $\hat{u}(k) \leq|\omega|_{\infty}+R_{0} \phi_{1}(k) \leq\left(|\omega|_{\infty}+R_{0}\right)\left(1+\phi_{1}(k)\right)$ and $c \phi_{1}(k) \leq \bar{u}(k)$ (see Lemma 2.11), the estimates asserted in the theorem follow.

\section{Acknowledgment}

This research was supported by National Natural Science Foundation (NNSF) of China (10301033).

\section{References}

[1] R. P. Agarwal, Difference Equations and Inequalities, 2nd ed., Monographs and Textbooks in Pure and Applied Mathematics, vol. 228, Marcel Dekker, New York, 2000.

[2] R. P. Agarwal, D. O'Regan, and P. J. Y. Wong, Positive Solutions of Differential, Difference and Integral Equations, Kluwer Academic, Dordrecht, 1999.

[3] F. Merdivenci Atici, Existence of positive solutions of nonlinear discrete Sturm-Liouville problems, Mathematical and Computer Modelling 32 (2000), no. 5-6, 599-607.

Haishen Lü: Department of Applied Mathematics, College of Sciences, Hohai University,

Nanjing 210098, China

E-mail address: haishen2001@yahoo.com.cn

Donal O'Regan: Department of Mathematics, Faculty of Science, National University of Ireland, Galway, University Road, Galway, Ireland

E-mail address: donal.oregan@nuigalway.ie

Ravi P. Agarwal: Department of Mathematical Sciences, College of Science,

Florida Institute of Technology, Melbourne, FL 32901-6975, USA

E-mail address: agarwal@fit.edu 


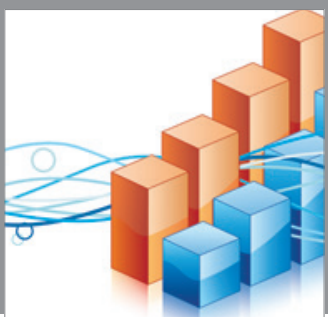

Advances in

Operations Research

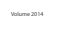

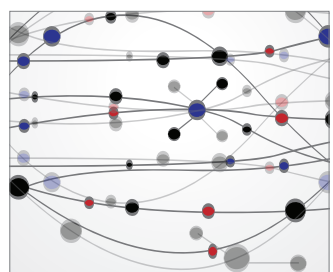

\section{The Scientific} World Journal
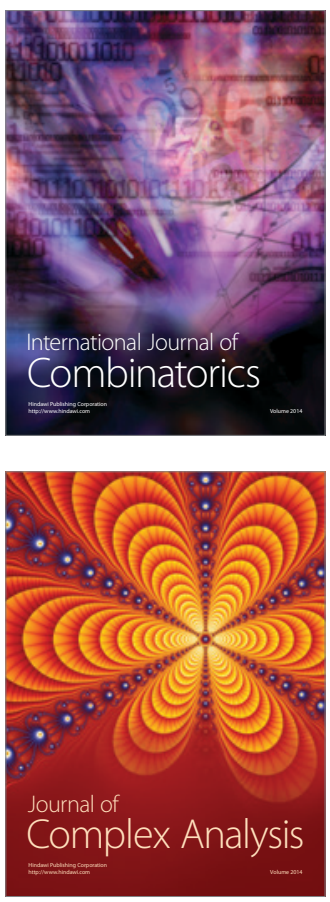

International Journal of

Mathematics and

Mathematical

Sciences
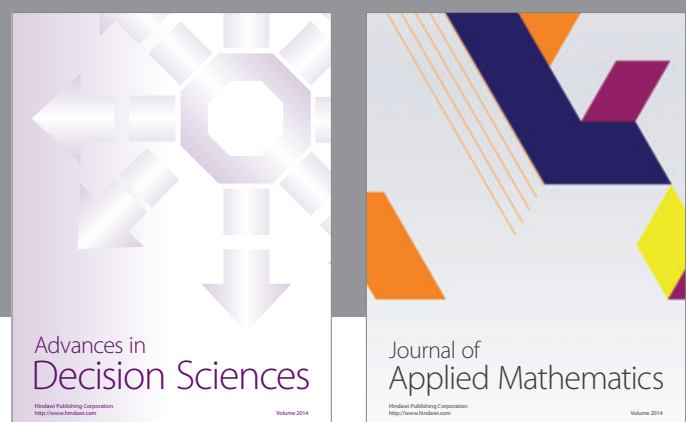

Journal of

Applied Mathematics
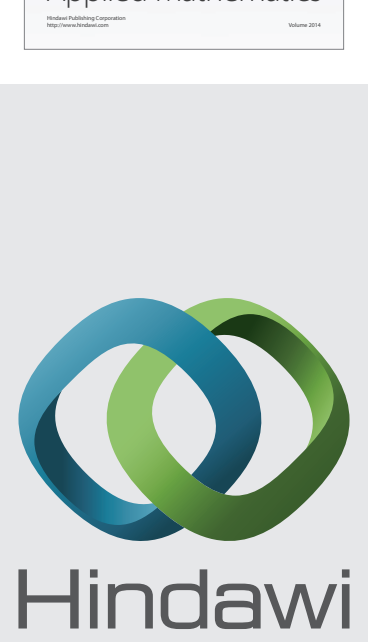

Submit your manuscripts at http://www.hindawi.com
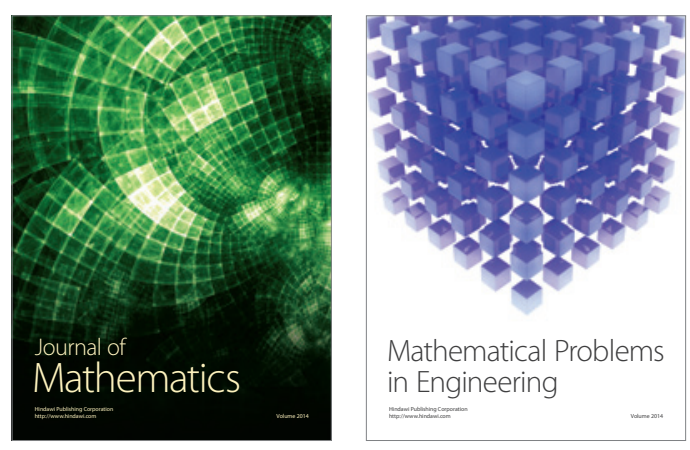

Mathematical Problems in Engineering
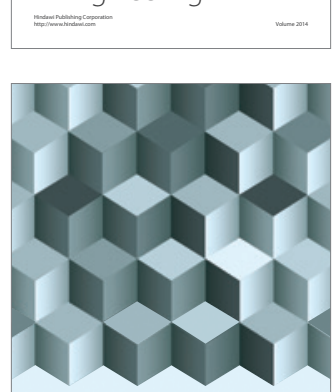

Journal of

Function Spaces
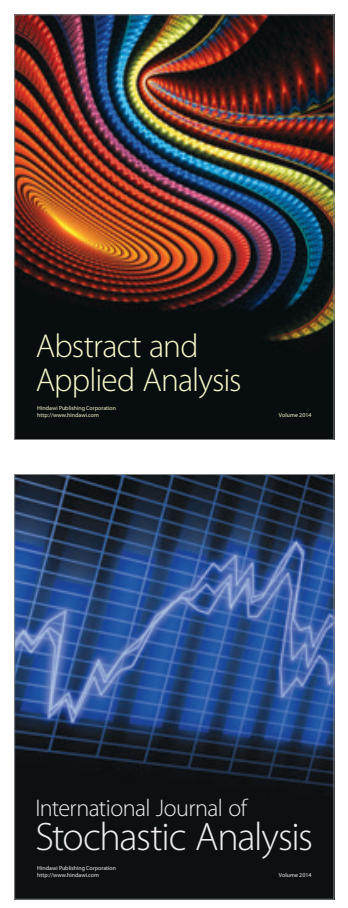

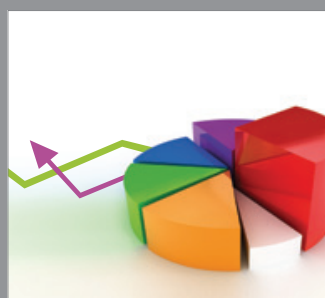

ournal of

Probability and Statistics

Promensencen
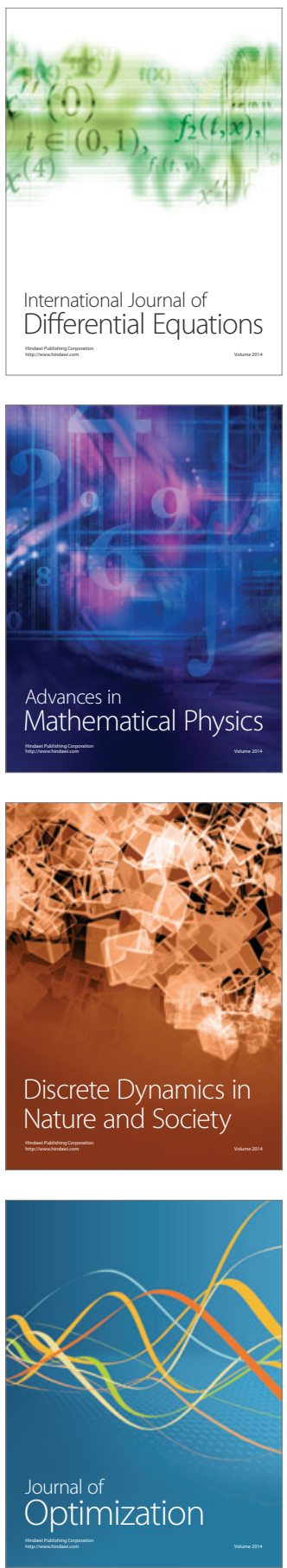